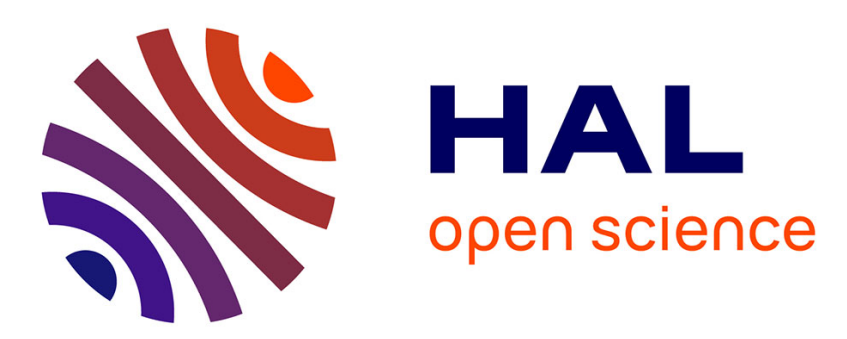

\title{
Remainder Padé approximants for hypergeometric series
} Marc Prévost, Tanguy Rivoal

\section{To cite this version:}

Marc Prévost, Tanguy Rivoal. Remainder Padé approximants for hypergeometric series. 2019. hal02017857

\section{HAL Id: hal-02017857 https://hal.science/hal-02017857}

Preprint submitted on 13 Feb 2019

HAL is a multi-disciplinary open access archive for the deposit and dissemination of scientific research documents, whether they are published or not. The documents may come from teaching and research institutions in France or abroad, or from public or private research centers.
L'archive ouverte pluridisciplinaire HAL, est destinée au dépôt et à la diffusion de documents scientifiques de niveau recherche, publiés ou non, émanant des établissements d'enseignement et de recherche français ou étrangers, des laboratoires publics ou privés. 


\title{
REMAINDER PADÉ APPROXIMANTS FOR HYPERGEOMETRIC SERIES
}

\author{
MARC PRÉVOST AND TANGUY RIVOAL
}

\begin{abstract}
Remainder Padé Approximation (RPA) consists in adding to the $n$-th partial sum of a series a suitable Padé approximant of the asymptotic expansion in the variable $1 / n$ of the remainder term. In a previous paper, we proved the non-trivial property that the RPA of the exponential function is identical to the Padé approximant to the function $e^{z}$. In this paper, we extend this property to the hypergeometric series ${ }_{1} F_{1}(1 ; a ; z)$ and ${ }_{2} F_{0}(b, 1 ; z)$.
\end{abstract}

\section{INTRODUCTION}

1.1. Remainder Padé Approximants. In [5], the first author introduced a new kind of rational approximation, the Remainder Padé Approximants (RPA). They are defined as follows. Let $f(z)=\sum_{k=0}^{\infty} a_{k} z^{k} \in \mathbb{C}[[z]]$; for simplicity of the exposition, in the discussion below $f$ is assumed to be convergent in a disk $D$ of positive radius $\left({ }^{1}\right)$. For any integer $n \geq 0$, we assume the existence of certain functions $b_{n}(z) \in \mathbb{C}(z)$ such that the normalized remainder series $\Phi(z, n):=\frac{1}{b_{n}(z)} \sum_{k=n}^{\infty} a_{k} z^{k}$ admits, for any fixed $z \in D \backslash\left\{\right.$ zeros of all the $\left.b_{n}\right\}$, an asymptotic expansion as $n \rightarrow+\infty$ of the form

$$
\Phi(z, n) \sim \widehat{\Phi}_{z}\left(\frac{1}{n}\right):=\sum_{k=0}^{\infty} \frac{\phi_{k}(z)}{n^{k}} \in \mathbb{C}(z)\left[\left[\frac{1}{n}\right]\right] .
$$

Let $[p / q]_{\widehat{\Phi}_{z}(t)}$ denote the ordinary Padé approximant of degrees $p$ and $q$ of the formal power series $\widehat{\Phi}_{z}(t)$ at $t=0$; it belongs to $\mathbb{C}(z, t)$. We thus have

$$
f(z)=\sum_{k=0}^{n-1} a_{k} z^{k}+b_{n}(z) \Phi_{n}(z) \approx \sum_{k=0}^{n} a_{k} z^{k}+b_{n}(z)\left([p / q]_{\widehat{\Phi}_{z}(t)}\right)_{t=1 / u(n)} .
$$

At this stage, the $\approx$ sign does not have any particular meaning, and the sequence of complex numbers $u(n)$ is $n+o(n)$ but not necessarily equal to $n$. We say that the rational fraction

$$
\frac{A(z)}{B(z)}:=\sum_{k=0}^{n-1} a_{k} z^{k}+b_{n}(z)\left([p / q]_{\widehat{\Phi}_{z}(t)}\right)_{t=1 / u(n)}
$$

2010 Mathematics Subject Classification. Primary 41A21; Secondary 41A28.

Key words and phrases. Remainder Padé approximants, hypergeometric function, multiple Charlier polynomials.

${ }^{1}$ It is possible to extend the notion of RPA when $f$ diverges everywhere but is summable in some sense; we present an exemple of this situation in Theorem 2 . 
is a Remainder Padé Approximant of $f(z)$, where $A(z), B(z) \in \mathbb{C}[z]$ are of lowest degrees. In certain cases, there exists a sequence $u(n)$ such that the corresponding RPA of $f(z)$ coincides with an ordinary Padé approximant of $f(z)$ at $z=0$, say $[N / D]_{f(z)}$. It is not yet understood for which class of functions $f$ this remarkable fact happens. When it does happen, the $\approx \operatorname{sign}$ in (1.1) can then be understood as follows: the order at $z=0$ of the remainder $B(z) f(z)-A(z)$ is larger than or equal to $N+D+1$, the order of Padé approximation.

More generally, it also happens that, instead of Padé approximants, we recover some previsously known functional approximations of $f(z)$, or even numerical approximations of certain of its values. For instance, the first author constructed certain sequences of $\operatorname{RPA}$ for $\zeta(2)=\sum_{k=1}^{\infty} 1 / k^{2}$ and $\zeta(3)=\sum_{k=1}^{\infty} 1 / k^{3}$ (both can be viewed as values of the polylogarithms $\sum_{k=1}^{\infty} z^{k} / k^{s}$ at $\left.z=1\right)$ and proved that they are exactly the sequences of rationals numbers used by Apéry [2] to prove the irrationality of $\zeta(2)$ and $\zeta(3)$. In [7], the second author used a modification of these RPA to produce a sequence of fast converging rational approximations for Catalan's constant $G=\sum_{k=0}^{\infty}(-1)^{k} /(2 k+1)^{2}$. In [6], using certain discrete multiple orthogonal polynomials introduced in [3], which generalize the classical Charlier orthogonal polynomials, we proved that certain RPA of $e^{z}$ provide the Padé approximants of the function $e^{z}$.

All these examples provide various meanings to the $\approx$ sign in $(1.1)$ and we use the vocable RPA phenomenon to cover such non-trivial properties.

We recall the notion of type II (diagonal) Padé approximants. For a given family $\left(F_{j}(X)\right)_{j=1, \ldots, r}$ of formal series in $\mathbb{C}[[X]]$ and any integers $p$ and $q$ such that $p \geq(r-1) q \geq 0$, there exist (by linear algebra) some polynomials $P_{1}(x), \ldots, P_{r}(x)$ and $Q(X)$ in $\mathbb{C}[X]$, not all 0 , such that $\operatorname{deg}\left(P_{j}\right) \leq p, \operatorname{deg}(Q) \leq r \cdot q$ and

$$
Q(X) F_{j}(X)-P_{j}(X)=\mathcal{O}\left(X^{p+q+1}\right), \quad 1 \leq j \leq r .
$$

The fractions $P_{j} / Q$ are unique and are by definition the type II Padé approximants of $\left(F_{j}(X)\right)_{j=1, \ldots, r}$ of parameters $(p, q)$. When $r=1$, we recover the ordinary Padé approximants $[p / q]$ of $F_{1}(X)$.

1.2. The results. The goal of this paper is to show that the RPA phenomenon occurs (in various generality) for the hypergeometric series

$$
E_{a}(z):={ }_{1} F_{1}(1 ; a ; z)=\sum_{k=0}^{\infty} \frac{z^{k}}{(a)_{k}} \quad \text { and } \quad{ }_{2} F_{0}(b, 1 ; z)=\sum_{k=0}^{\infty}(b)_{k} z^{k} .
$$

We assume that $a \in \mathbb{C} \backslash \mathbb{Z}_{\leq 0}$. The former series generalizes the exponential function ${ }_{1} F_{1}(1 ; 1 ; z)=\exp (z)$, while the latter generalizes Euler's series ${ }_{2} F_{0}(1,1 ; z)=\sum_{k=0}^{\infty} k ! z^{k}$. The variable $z$ is a formal one in Theorems 1 and 2 below. However, it is important to have in mind that, though the series $E_{a}(z)$ is an entire function of $z$, the ${ }_{2} F_{0}(b, 1 ; z)$ diverges for every $z$. To overcome this divergence, we shall first work the function

$$
\mathcal{E}_{b}(z):=\frac{1}{\Gamma(b)} \int_{0}^{\infty} \frac{u^{b-1}}{1-u z} e^{-u} d u
$$


defined for $\Re(b)>0$ and analytic for $z \in \mathbb{C} \backslash[0,+\infty)$. It admits an asymptotic expansion $\widehat{\mathcal{E}}_{b}(z)$ as $z \rightarrow 0$ in any angular sector centered at 0 that does not contain $[0,+\infty)$. It turns out that $\widehat{\mathcal{E}}_{b}(z)={ }_{2} F_{0}(b, 1 ; z)$ in such sectors.

To state our results, we need to introduce a certain number of notations. The parameters $\alpha_{j}, j=1, \ldots, r$, are pairwise distinct non-zero complex numbers, and we set $\underline{\alpha}:=\left(\alpha_{j}\right)_{j=1, \ldots, r}$. For any $z \in \mathbb{C}$ and any $t \in \mathbb{C}$ such that $1 / t \notin \mathbb{N}$, we define

$$
\Phi_{z}(t):=\sum_{k=0}^{\infty} \frac{z^{k}}{(1-1 / t)_{k}} .
$$

For any $j=1, \ldots, r$ and any $n \geq 0$, we have the trivial identity

$$
\sum_{k=0}^{\infty} \frac{\left(\alpha_{j} z\right)^{k}}{(a)_{k}}=\sum_{k=0}^{n-1} \frac{\left(\alpha_{j} z\right)^{k}}{(a)_{k}}+\frac{\left(\alpha_{j} z\right)^{n}}{(a)_{n}} \Phi_{\alpha_{j} z}\left(\frac{-1}{a-1+n}\right) .
$$

In [6], it has been proved that, for any fixed $z \in \mathbb{C}, \Phi_{z}(t)$ admits an asymptotic expansion $\widehat{\Phi}_{z}(t):=\sum_{k=0}^{\infty} \varphi_{k}(-z) t^{k}$ as $t \rightarrow 0, t<0$, where the coefficients $\varphi_{k}(z)$ are Touchard exponential polynomials of degree $k$. The latter are defined by the exponential generating function $e^{z\left(e^{X}-1\right)}=\sum_{k=0}^{\infty} \varphi_{k}(z) \frac{X^{k}}{k !}$ (see [10]).

We provided in [6] explicit expressions for the type II Padé approximants at $t=0$ of parameters $(r p-1, p)$ for the formal power series $\widehat{\Phi}_{\alpha_{j} z}(t)$ in $\mathbb{C}[z][[t]]$ for $j=1, \ldots, r$ : we denote them by $P_{j, \underline{\alpha}, p}(t, z) / Q_{\underline{\alpha}, p}(t, z)$, and the expressions of these polynomials in $t$ are given in $\S 2$. It turns out that they are also polynomials in $z$.

We now "replace" $\Phi_{\alpha_{j} z}\left(\frac{-1}{a-1+n}\right)$ in (1.2) by the corresponding simultaneous Padé approximant of parameters $(r p-1, p)$ of $\widehat{\Phi}_{\alpha_{j} z}(t)$ evaluated at $t=-1 /(a-1+n)$ (this substitution is possible because $Q_{\underline{\alpha}, p}(-1 /(a-1+n), z)$ does not vanish identically as a function of $\left.z\right)$. This alteration of (1.2) provides the formal Laurent series

$$
R_{j, n, \underline{\alpha}, p}(z):=\sum_{k=0}^{\infty} \frac{\left(\alpha_{j} z\right)^{k}}{(a)_{k}}-\sum_{k=0}^{n-1} \frac{\left(\alpha_{j} z\right)^{k}}{(a)_{k}}-\frac{\left(\alpha_{j} z\right)^{n}}{(a)_{n}} \frac{P_{j, \underline{\alpha}, p}\left(\frac{-1}{a-1+n}, z\right)}{Q_{\underline{\alpha}, p}\left(\frac{-1}{a-1+n}, z\right)}, \quad j=1, \ldots, r,
$$

and we want to know in which sense they are $\approx 0$. By construction, for every $j$, the rational fraction

$$
\sum_{k=0}^{n-1} \frac{\left(\alpha_{j} z\right)^{k}}{(a)_{k}}+\frac{\left(\alpha_{j} z\right)^{n}}{(a)_{n}} \frac{P_{j, \underline{\alpha}, p}\left(\frac{-1}{a-1+n}, z\right)}{Q_{\underline{\alpha}, p}\left(\frac{-1}{a-1+n}, z\right)}
$$

is a RPA of $E_{a}\left(\alpha_{j} z\right)$. The degrees of its numerator and denominator are a priori bounded by $n+r p-1$ and $r p$ respectively. A better bound holds for the numerator $((i)$ below $)$ and it is crucial to prove $(i i)$. (Throughout the paper, $\mathcal{O}\left(z^{c}\right)$ denotes a Laurent series in $z$ with order equal to $c$ at $z=0$.)

Theorem 1. Let us fix the integers $r \geq 1, n \geq 1, p \geq 0$, such that $n \geq p, a \in \mathbb{C} \backslash \mathbb{Z}_{\leq 0}$, $\alpha_{1}, \ldots, \alpha_{r} \in \mathbb{C}$ pairwise distinct. 
(i) The degrees of the numerator and denominator of the RPA in (1.3) are bounded by $n+(r-1) p-1$ and rp respectively, and moreover $R_{j, n, \underline{\alpha}, p}(z)=\mathcal{O}\left(z^{n+r p}\right)$.

(ii) The collection of the RPA in (1.3), $j=1, \ldots, r$, is the type II Padé approximant of $\left(E_{a}\left(\alpha_{j} z\right)\right)_{j=1, \ldots, r}$ of parameters $(n+(r-1) p-1, p)$.

(iii) For $r=1$ and $\alpha_{1}=1$, (ii) gives the following equality of rational fractions:

$$
\sum_{k=0}^{n-1} \frac{z^{k}}{(a)_{k}}+\frac{z^{n}}{(a)_{n}}\left([p-1 / p]_{\widehat{\Phi}_{z}(t)}\right)_{t=-1 /(a-1+n)}=[n-1 / p]_{E_{a}(z)},
$$

For a generic value of $a$, our proof needs the assumption that $n \geq p$. However, if $a=1$, it can be removed in the statement of Theorem 1 , and we simply recover the results from [6]. The explanation of this fact is Identity (2.5) in $\S 2$.

Let us now describe our results for the function $\mathcal{E}_{b}(z)$. For any $n \geq 0, b$ such that $\Re(b)>0$ and $z \in \mathbb{C} \backslash[0,+\infty)$, we have the identity (proved in $\S 3$ )

$$
\mathcal{E}_{b}(z)=\sum_{k=0}^{n-1}(b)_{k} z^{k}+(b)_{n} z^{n} \Psi_{z}\left(\frac{1}{b-1+n}\right)
$$

where

$$
\Psi_{z}(t)=\frac{1}{\Gamma(1+1 / t)} \int_{0}^{\infty} \frac{u^{1 / t}}{1-u z} e^{-u} d u
$$

is defined for any $z \in \mathbb{C} \backslash(0,+\infty)$ and any $t$ such that $\Re(1 / t)>-1$ (in particular, for any $t>0)$. We shall prove in $\S 3$ that when $z<0, \Psi_{z}(t)$ admits an asymptotic expansion as $t \rightarrow 0, t>0$, given by $\left({ }^{2}\right)$

$$
\widehat{\Psi}_{z}(t):=-\frac{1}{z} \sum_{k=0}^{\infty} \varphi_{k}(1 / z) t^{k+1}=-\frac{t}{z} \widehat{\Phi}_{-1 / z}(t) .
$$

It then immediately follows that the Padé approximant $[p / p]$ at $t=0$ of the formal power series $\widehat{\Psi}_{z}(t) \in \mathbb{C}[1 / z][[t]]$ is $-\frac{t}{z} \frac{P_{j, 1, p}(t,-1 / z)}{Q_{1, p}(t,-1 / z)}$ where the polynomials are those used to construct the Padé approximants $[p-1 / p]$ of $\widehat{\Phi}_{z}(t)$ when $r=1$.

In (1.4), we now "replace" $\mathcal{E}_{b}(z)$ by $\widehat{\mathcal{E}}_{b}(z)$, and $\Psi_{z}\left(\frac{1}{b-1+n}\right)$ by the Padé approximant $[p / p]$ of $\widehat{\Psi}_{z}(t)$ evaluated at $t=1 /(b-1+n)$ (this subtitution is possible because $Q_{1, p}(1 /(b-1+$ $n), 1 / z)$ does not vanish identically as a function of $z$ ). This alteration of (1.4) provides the formal Laurent series

$$
S_{n, p}(z):=\widehat{\mathcal{E}}_{b}(z)-\sum_{k=0}^{n-1}(b)_{k} z^{k}+\frac{(b)_{n} z^{n-1}}{(b-1+n)} \frac{P_{j, 1, p}\left(\frac{1}{b-1+n},-\frac{1}{z}\right)}{Q_{1, p}\left(\frac{1}{b-1+n},-\frac{1}{z}\right)},
$$

\footnotetext{
${ }^{2}$ This identity is reminiscent of André's duality between solutions of $E$-operators at $z=0$ and $z=\infty$; see [1]. The former involve function like $E_{a}(z)$, while the latter involve functions like $\widehat{\mathcal{E}}_{b}(1 / z)$, with $a, b \in \mathbb{Q}$.
} 
and we want to know in which sense it is $\approx 0$. Because of $(1.4)$, the rational fraction

$$
\sum_{k=0}^{n-1}(b)_{k} z^{k}-\frac{(b)_{n} z^{n-1}}{(b-1+n)} \frac{P_{j, 1, p}\left(\frac{1}{b-1+n},-\frac{1}{z}\right)}{Q_{1, p}\left(\frac{1}{b-1+n},-\frac{1}{z}\right)}
$$

can be viewed as a RPA of $\mathcal{E}_{b}(z)$ in an extended sense. The degrees of its numerator and denominator are a priori bounded by $n+p-1$ and $p$ respectively. Again, a better bound holds for the numerator $((i)$ below) and again it is crucial to prove (ii).

Theorem 2. Let us fix $b \in \mathbb{C} \backslash \mathbb{Z}_{\leq 0}$, and the integers $n \geq 1, p \geq 0$ such that $n \geq p$.

(i) The degrees of the numerator and denominator of the RPA in (1.6) are bounded by $n-1$ and $p$ respectively, and moreover $S_{n, p}(z)=\mathcal{O}\left(z^{n+p}\right)$ exists and is finite.

(ii) The RPA in (1.6) coincides with the Padé approximant $[n-1 / p]$ of $\widehat{\mathcal{E}}_{b}(z)$. In other words,

$$
\sum_{k=0}^{n-1}(b)_{k} z^{k}+(b)_{n} z^{n}\left([p / p]_{\widehat{\Psi}_{z}(t)}\right)_{t=1 /(b-1+n)}=[n-1 / p]_{\widehat{\mathcal{E}}_{b}(z)} .
$$

It seems that a RPA phenomenom similar to $(i i)$ in Theorem 1 does not hold for any family $\left(\widehat{\mathcal{E}}_{b}\left(\beta_{j}\right)\right)_{j=1, \ldots, r}$ with $r \geq 2$.

The proofs of Theorems 1 and 2 are given in $\S 2$ and $\S 3$ respectively.

\section{Proof of TheOrem 1}

Proof of $(i)$. We recall the expressions of the type II approximants of the functions $\widehat{\Phi}_{\alpha_{j} z}(t)$ (obtained in $[6]$ ):

$$
\begin{aligned}
& P_{j, \underline{\alpha}, p}(t, z)= \\
& \quad(-t)^{r p-1} \sum_{k_{1}, \ldots, k_{r}=0}^{p}\left(\prod_{i=1}^{r}\left(\begin{array}{l}
p \\
k_{i}
\end{array}\right)\left(-\alpha_{i} z\right)^{p-k_{i}}\right)(-1 / t)_{k_{1}+\cdots+k_{r}} \sum_{i=1}^{k_{1}+\cdots+k_{r}} \frac{\left(\alpha_{j} z\right)^{i-1}}{(-1 / t)_{i}},
\end{aligned}
$$

and

$$
Q_{\underline{\alpha}, p}(t, z)=(-t)^{r p} \sum_{k_{1}, \ldots, k_{r}=0}^{p}\left(\prod_{i=1}^{r}\left(\begin{array}{c}
p \\
k_{i}
\end{array}\right)\left(-\alpha_{i} z\right)^{p-k_{i}}\right)(-1 / t)_{k_{1}+\cdots+k_{r}} .
$$

The $P$ 's have degree $r p-1$ in both $t$ and $z$, while the $Q$ 's have degree $r p$ in both $t$ and $z$. Moreover, $Q_{\underline{\alpha}, p}(0,1)=1$.

For the sake of simplicity, we set $K_{r}=k_{1}+k_{2}+\cdots+k_{r}$. Substituting $-1 /(a-1+n)$ for $t$ (which is possible because $a \notin \mathbb{Z}_{\leq 0}$ and $n \geq 1$ ), we get

$$
Q_{\underline{\alpha}, p}\left(\frac{-1}{a-1+n}, z\right)=(a+n-1)^{-r p} \sum_{k_{1}, \ldots, k_{r}=0}^{p}\left(\prod_{i=1}^{r}\left(\begin{array}{c}
p \\
k_{i}
\end{array}\right)\left(-\alpha_{i} z\right)^{p-k_{i}}\right)(a-1+n)_{K_{r}}
$$


and

$$
\begin{aligned}
P_{j, \underline{\alpha}, p}\left(\frac{-1}{a-1+n}, z\right) & =\frac{(a-1+n)^{1-r p}(a)_{n-1}}{\left(\alpha_{j} z\right)^{n}} \\
\times \sum_{k_{1}, \ldots, k_{r}=0}^{p} & \left(\prod_{i=1}^{r}\left(\begin{array}{c}
p \\
k_{i}
\end{array}\right)\left(-\alpha_{i} z\right)^{p-k_{i}}\right)(a-1+n)_{K_{r}}\left(S_{n+K_{r}-1}\left(\alpha_{j} z\right)-S_{n-1}\left(\alpha_{j} z\right)\right)
\end{aligned}
$$

where $S_{n}(z)=\sum_{k=0}^{n} z^{k} /(a)_{k}$. (Note that $Q_{\underline{\alpha}, p}\left(\frac{-1}{a-1+n}, z\right)$ is clearly not identically zero, as stated in the Introduction.)

Hence, after some simplifications, the RPAs of the functions $E_{\alpha_{j}}(z), j=1, \ldots, r$, are given by

$$
\begin{aligned}
\sum_{k=0}^{n-1} \frac{\left(\alpha_{j} z\right)^{k}}{(a)_{k}}+\frac{\left(\alpha_{j} z\right)^{n}}{(a)_{n}} & \frac{P_{j, \underline{\alpha}, p}\left(\frac{-1}{a-1+n}, z\right)}{Q_{\underline{\alpha}, p}\left(\frac{-1}{a-1+n}, z\right)} \\
= & \frac{\sum_{k_{1}, \ldots, k_{r}=0}^{p}\left(\prod_{i=1}^{r}\left(\begin{array}{c}
p \\
k_{i}
\end{array}\right)\left(-\alpha_{i} z\right)^{p-k_{i}}\right)(a-1+n)_{K_{r}} S_{n+K_{r}-1}\left(\alpha_{j} z\right)}{\sum_{k_{1}, \ldots, k_{r}=0}^{p}\left(\prod_{i=1}^{r}\left(\begin{array}{c}
p \\
k_{i}
\end{array}\right)\left(-\alpha_{i} z\right)^{p-k_{i}}\right)(a-1+n)_{K_{r}}} .
\end{aligned}
$$

At first sight, the numerator and denominator of this rational fraction have degrees bounded by $n+r p-1$ and $r p$ in $z$ respectively, but a better estimate holds for the numerator. Indeed, we shall now prove that its degree is bounded by $n+(r-1) p-1$. Indeed, with $K_{j, r}^{\prime}=k_{1}+\cdots+k_{j-1}+k_{j+1}+\cdots+k_{r}$ and $\underline{k}_{j, r}^{\prime}=\left(k_{1}, \ldots, k_{j-1}, k_{j+1}, \ldots, k_{r}\right)$, the numerator of (2.3) can be expressed as

$$
\sum_{k_{1}, \ldots, k_{j-1}, k_{j+1}, \ldots, k_{r}=0}^{p}\left(\prod_{i=1, i \neq j}^{r}\left(\begin{array}{l}
p \\
k_{i}
\end{array}\right)\left(-\alpha_{i} z\right)^{p-k_{i}}\right)(a-1+n)_{K_{j, r}^{\prime}} A_{j, \underline{k}_{j, r}^{\prime}}(z)
$$

with

$$
A_{j, \underline{k}_{j, r}^{\prime}}(z)=\sum_{k_{j}=0}^{p}\left(\begin{array}{c}
p \\
k_{j}
\end{array}\right)\left(-\alpha_{j} z\right)^{p-k_{j}}\left(a-1+n+K_{j, r}^{\prime}\right)_{k_{j}} S_{n+K_{r}-1}\left(\alpha_{j} z\right) .
$$

We claim that the degree in $z$ of the polynomial $A_{j, \underline{k}_{j, r}^{\prime}}(z)$ is at $\operatorname{most} \max \left(p, n+K_{j, r}^{\prime}\right)-1$. To prove this observation, which is non-trivial, we first observe that, for any integers $m, q$ such that $m \geq q \geq 0$,

$$
\left(t^{a-1} S_{m}(x t)\right)_{\mid t=1}^{(q)}=x^{q} S_{m-q}(x)+U_{q}(x)
$$

where

$$
U_{q}(x):=\sum_{j=0}^{q-1}(-1)^{q-j}(1-a)_{q-j} x^{j}
$$


(Above and below in this section, all the differentiations are with respect to $t$.) Observe that $U_{q}(x) \equiv 0$ if $a=1$, but in general $U_{q}(x)$ has degree $q-1$ in $x$. Since $(a-1+m)_{k}=$ $(-1)^{k}\left(t^{-a+1-m}\right)_{\mid t=1}^{(k)}$, it follows that

$$
\begin{aligned}
A_{j, \underline{k}_{j, r}^{\prime}}(z)=( & -1)^{p} \sum_{k=0}^{p}\left(\begin{array}{l}
p \\
k
\end{array}\right)\left(t^{-a+1-n-K_{j, r}^{\prime}}\right)_{\mid t=1}^{(k)}\left(t^{a-1} S_{n+K_{j, r}^{\prime}+p-1}\left(\alpha_{j} z t\right)\right)_{\mid t=1}^{(p-k)} \\
& +\sum_{k=0}^{p}(-1)^{p-k}\left(\begin{array}{l}
p \\
k
\end{array}\right)\left(a-1+n+K_{j, r}^{\prime}\right)_{k} U_{p-k}\left(\alpha_{j} z\right) \\
= & (-1)^{p}\left(t^{-n-K_{j, r}^{\prime}} S_{n+K_{j, r}^{\prime}+p-1}\left(\alpha_{j} z t\right)\right)_{\mid t=1}^{(p)} \\
& +\sum_{k=0}^{p}(-1)^{p-k}\left(\begin{array}{l}
p \\
k
\end{array}\right)\left(a-1+n+K_{j, r}^{\prime}\right)_{k} U_{p-k}\left(\alpha_{j} z\right) .
\end{aligned}
$$

The polynomial in (2.7) obviously has degree at most $p-1$ in $z$. On the other hand

$$
\begin{aligned}
\left(t^{-n-K_{j, r}^{\prime}} S_{n+K_{j, r}^{\prime}+p-1}\left(\alpha_{j} z t\right)\right)_{\mid t=1}^{(p)} & =\sum_{j=0}^{n+K_{j, r}^{\prime}+p-1} \frac{\left(\alpha_{j} z\right)^{j}}{(a)_{j}}\left(t^{j-n-K_{j, r}^{\prime}}\right)_{\mid t=1}^{(p)} \\
& =(-1)^{p} \sum_{j=0}^{n+K_{j, r}^{\prime}+p-1} \frac{\left(\alpha_{j} z\right)^{j}}{(a)_{j}}\left(n+K_{j, r}^{\prime}-j\right)_{p} .
\end{aligned}
$$

Since $\left(n+K_{j, r}^{\prime}-j\right)_{p}=0$ for any $j \in\left\{n+K_{j, r}^{\prime}, \ldots, n+K_{j, r}^{\prime}+p-1\right\}$, it follows that the polynomial in (2.6) has degree at most $n+K_{j, r}^{\prime}-1$, so that the degree of $A_{j, \underline{k}_{j, r}^{\prime}}(z)$ is at $\operatorname{most} \max \left(p, n+K_{j, r}^{\prime}\right)-1$ as claimed. Since $n \geq p$ by assumption, we deduce that the degree of the numerator of the RPAs given by $(2.4)$ is at most $n+(r-1) p-1$, as expected.

To prove that $R_{j, n, \underline{\alpha}, p}(z)=\mathcal{O}\left(z^{n+r p}\right)$, we have to multiply the denominator of $(2.3)$ by the series $\sum_{k=0}^{\infty} \frac{\left(\alpha_{j} z\right)^{k}}{(a)_{k}}$ and prove that the product is the sum of a polynomial of degree $n+(r-1) p-1$ and a function which is $\mathcal{O}\left(z^{n+r p}\right)$. This amounts to proving that the coefficient $c_{m}$ of $z^{m}$ in the Taylor expansion of

$$
\left(\sum_{k_{1}, \ldots, k_{r}=0}^{p}\left(\prod_{i=1}^{r}\left(\begin{array}{l}
p \\
k_{i}
\end{array}\right)\left(-\alpha_{i} z\right)^{p-k_{i}}\right)(a-1+n)_{K_{r}}\right) \cdot \sum_{k=0}^{\infty} \frac{\left(\alpha_{j} z\right)^{k}}{(a)_{k}}
$$

is zero for $m \in\{n+(r-1) p, \ldots, n+r p-1\}$. Let us assume that $m \geq n+(r-1) p$. Then

$$
c_{m}=\sum_{k_{1}, \ldots, k_{r}=0}^{p}\left(\prod_{i=1}^{r}\left(\begin{array}{l}
p \\
k_{i}
\end{array}\right)\left(-\alpha_{i}\right)^{p-k_{i}}\right)(a-1+n)_{K_{r}} \frac{\alpha_{j}^{k}}{(a)_{k}}
$$

where in (2.8) the integer $k:=m+K_{r}-r p$ is $\geq 0$ (because $m \geq n+(r-1) p \geq r p$ ). 
We now also assume that $m \leq n+r p-1$. In that case, we use the identity

$$
\frac{(a-1+n)_{K_{r}}}{(a)_{k}}=\frac{1}{(a)_{n-1}}\left(t^{a+n+K_{r}-2}\right)_{\mid t=1}^{(n+r p-m-1)}
$$

in $(2.8)$ and get

$$
\begin{aligned}
c_{m} & =\frac{1}{(a)_{n-1}} \sum_{k_{1}, \ldots, k_{r}=0}^{p}\left(\prod_{i=1}^{r}\left(\begin{array}{l}
p \\
k_{i}
\end{array}\right)\left(-\alpha_{i}\right)^{p-k_{i}}\right) \alpha_{j}^{m-r p+K_{r}}\left(t^{a+n+K_{r}-2}\right)_{\mid t=1}^{(n+r p-m-1)} \\
& =\frac{1}{(a)_{n-1}}\left(t^{a-1+n-r p} \prod_{i=1}^{r}\left(\alpha_{j}-\alpha_{i} t\right)^{p}\right)_{\mid t=1}^{(n+r p-m-1)} .
\end{aligned}
$$

Since $t=1$ is a zero of order $p$ of the product $\prod_{i=1}^{r}\left(\alpha_{j}-\alpha_{i} t\right)^{p}$, it follows that $c_{m}=0$ when $n+r p-m-1 \leq p-1$, i.e when $m \geq n+(r-1) p$. This complete the proof of $(i)$.

Proof of $(i i)$. By unicity of type II Padé approximants, the bounds for the degrees of the simultaneous RPAs and the order of $R_{j, n, \underline{\alpha}, p}$ at 0 ensure that (2.3) provides the expression of the type II Padé approximants of the family $\left(E_{a}\left(\alpha_{j} z\right)\right)_{j=1, \ldots, r}$ of parameters $(n+(r-1) p-1, p)$ when $n \geq p$.

\section{Proof of Theorem 2}

Let $b$ such that $\Re(b)>0$. We first prove (1.4) of the Introduction, i.e. that for any $n \geq 0$ and any $z \in \mathbb{C} \backslash[0,+\infty)$,

$$
\mathcal{E}_{b}(z)=\sum_{k=0}^{n-1}(b)_{k} z^{k}+(b)_{n} z^{n} \Psi_{z}\left(\frac{1}{b-1+n}\right) .
$$

This follows from

$$
\begin{aligned}
(b)_{n} z^{n} \Psi_{z}\left(\frac{1}{b-1+n}\right) & =\frac{(b)_{n} z^{n}}{\Gamma(b+n)} \int_{0}^{\infty} \frac{u^{b+n-1}}{1-u z} e^{-u} d u \\
& =\frac{(b)_{n}}{\Gamma(b+n)} \int_{0}^{\infty} \frac{u^{b-1}}{1-u z} e^{-u} d u+\frac{(b)_{n}}{\Gamma(b+n)} \int_{0}^{\infty} u^{b-1} \frac{(u z)^{n}-1}{1-u z} e^{-u} d u \\
& =\mathcal{E}_{b}(z)-\frac{(b)_{n}}{\Gamma(b+n)} \sum_{k=0}^{n-1} z^{k} \int_{0}^{\infty} u^{k+b-1} e^{-u} d u \\
& =\mathcal{E}_{b}(z)-\sum_{k=0}^{n-1} \frac{(b)_{n} \Gamma(b+k)}{\Gamma(b+n)} z^{k}=\mathcal{E}_{b}(z)-\sum_{k=0}^{n-1}(b)_{k} z^{k} .
\end{aligned}
$$

We now prove the following lemma.

Lemma 1. Let us fix $z \in(-\infty, 0)$. The function $\Psi_{z}(t)$ admits an asymptotic expansion as $t \rightarrow 0, t>0$, given by

$$
\widehat{\Psi}_{z}(t):=-\frac{1}{z} \sum_{k=0}^{\infty} \varphi_{k}(1 / z) t^{k+1}=-\frac{t}{z} \widehat{\Phi}_{-1 / z}(t),
$$


where the coefficients $\varphi_{k}(X)$ are Touchard exponential polynomials of degree $k$ in $X$.

Proof. We first use Proposition 2 in [8] to get the following alternative expression for $\Psi_{z}(t)$ : for any $z \in(-\infty, 0)$ and $t>0$,

$$
\Psi_{z}(t)=\int_{0}^{\infty} \frac{e^{-u}}{(1-z u)^{1+1 / t}} d u .
$$

We now set $v=\ln (1-u z)$, so that

$$
\Psi_{z}(t)=-\frac{1}{z} \int_{0}^{\infty} e^{\left(e^{v}-1\right) / z} e^{-v / t} \mathrm{~d} v
$$

for $z \in(-\infty, 0)$ and $t>0$. Since $v \mapsto e^{\left(e^{v}-1\right) / z}$ is $C^{\infty}$ at $v=0$, non-zero at $v=0$, and

$$
\int_{0}^{\infty}\left|e^{\left(e^{v}-1\right) / z} e^{-v / t}\right| \mathrm{d} v<\infty
$$

we can apply Watson's lemma $([4$, Chapter $2, \S 2])$ to $(3.1)$ : the function $\Psi_{z}(t)$ has the asymptotic expansion

$$
\Psi_{z}(t) \sim-\frac{1}{z} \sum_{k=0}^{\infty}\left(\frac{d^{k}}{d v^{k}} e^{\left(e^{v}-1\right) / z}\right)_{\mid v=0} t^{k+1}=-\frac{t}{z} \sum_{k=0}^{\infty} \varphi_{k}(1 / z) t^{k}, \quad t \rightarrow 0, t>0 .
$$

This complete the proof of the lemma.

From now on, we view $\widehat{\Psi}_{z}(t)$ as a formal series in $\mathbb{C}[1 / z][[t]]$. As observed in the Introduction, the identity $\widehat{\Psi}_{z}(t)=-\frac{t}{z} \widehat{\Phi}_{-1 / z}(t)$ enables us to immediately get the Padé approximant $[p / p]$ of $\widehat{\Psi}_{z}(t)$ at $t=0$. More generally, let $\beta:=\left(\beta_{j}\right)_{j=1, \ldots, r}$ denote a family of pairwise distinct complex numbers. Then, without further efforts, we even obtain the type II Padé approximants at $t=0$ of parameters $(r p, p)$ of the formal power series $\left(\widehat{\Psi}_{\beta_{j} z}(t)\right)_{j=1, \ldots, r}$ for $j=1, \ldots, r$. They are given by

$$
-\frac{t}{\beta_{j} z} \frac{P_{j, 1 / \underline{\beta}, p}(t,-1 / z)}{Q_{1 / \underline{\beta}, p}(t,-1 / z)}, \quad j=1, \ldots, r
$$

where

$$
\begin{aligned}
& P_{j, 1 / \underline{\beta}, p}(t,-1 / z)(-t)^{r p-1} \sum_{k_{1}, \ldots, k_{r}=0}^{p}\left(\prod_{i=1}^{r}\left(\begin{array}{c}
p \\
k_{i}
\end{array}\right)\left(\beta_{i} z\right)^{k_{i}-p}\right)(-1 / t)_{k_{1}+\cdots+k_{r}} \sum_{i=1}^{k_{1}+\cdots+k_{r}} \frac{\left(-\beta_{j} z\right)^{1-i}}{(-1 / t)_{i}}, \\
& Q_{1 / \underline{\beta}, p}(t,-1 / z)=(-t)^{r p} \sum_{k_{1}, \ldots, k_{r}=0}^{p}\left(\prod_{i=1}^{r}\left(\begin{array}{c}
p \\
k_{i}
\end{array}\right)\left(\beta_{i} z\right)^{k_{i}-p}\right)(-1 / t)_{k_{1}+\cdots+k_{r}} .
\end{aligned}
$$

We now substitute $1 /(b-1+n)$ for $t$ in these expressions, which is possible because $\Re(b)>0$ and $n \geq 1$. Since $Q_{1 / \beta, p}(1 /(b-1+n),-1 / z)$ is clearly not identically zero, we 
get simultaneous RPAs of the family $\left(\mathcal{E}_{b}\left(\beta_{j} z\right)\right)_{j=1, \ldots, r}$ given by the formulas:

$$
\begin{aligned}
\sum_{k=0}^{n-1}(b)_{k}\left(\beta_{j} z\right)^{k}-(b)_{n} \frac{\left(\beta_{j} z\right)^{n-1}}{(b-1+n)} \frac{P_{j, 1 / \underline{\beta}, p}\left(\frac{1}{b-1+n},-\frac{1}{z}\right)}{Q_{1 / \underline{\beta}, p}\left(\frac{1}{b-1+n},-\frac{1}{z}\right)} & z^{r p} \sum_{k_{1}, \ldots, k_{r}=0}^{p}\left(\prod_{i=1}^{r}\left(\begin{array}{c}
p \\
k_{i}
\end{array}\right)\left(\beta_{i} z\right)^{k_{i}-p}\right)(-1)^{K_{r}} T_{n-K_{r}-1}\left(\beta_{j} z\right) /(b)_{n-K_{r}} \\
= & z^{r p} \sum_{k_{1}, \ldots, k_{r}=0}^{p}\left(\prod_{i=1}^{r}\left(\begin{array}{l}
p \\
k_{i}
\end{array}\right)\left(\beta_{i} z\right)^{k_{i}-p}\right)(-1)^{K_{r}} /(b)_{n-K_{r}}
\end{aligned}
$$

where $T_{n}(z):=\sum_{k=0}^{n}(b)_{k} z^{k}$.

We are now in position to complete the proof of Theorem 2 . We take $r=1$ and $\beta_{1}=1$ in $(3.2)$.

Proof of $(i)$. It is immediate that the degrees of the numerator and denominator of the rational function in (3.2) are bounded by $n-1$ and $p$ respectively. We now want to prove that, formally,

$$
\widehat{\mathcal{E}}_{b}(z)-\sum_{k=0}^{n-1}(b)_{k} z^{k}+\frac{(b)_{n} z^{n-1}}{(b-1+n)} \frac{P_{j, 1, p}\left(\frac{1}{b-1+n},-\frac{1}{z}\right)}{Q_{1, p}\left(\frac{1}{b-1+n},-\frac{1}{z}\right)}=\mathcal{O}\left(z^{n+p}\right) .
$$

The denominator of the right-hand side of (3.2) is

$$
Q(z):=\sum_{k=0}^{p} \frac{(-1)^{k}\left(\begin{array}{l}
p \\
k
\end{array}\right)}{(b)_{n-k}} z^{k}
$$

so that to prove (3.3) it is enough to prove that the formal series expansion of

$$
\left(\sum_{k=0}^{p} \frac{(-1)^{k}\left(\begin{array}{l}
p \\
k
\end{array}\right)}{(b)_{n-k}} z^{k}\right) \widehat{\mathcal{E}}_{b}(z)
$$

is the sum of a polynomial $P(z)$ of degree $\leq n-1$ and a formal power series of order $\geq n+p$ at $z=0$. This in fact means that we want to prove that $P(z) / Q(z)$ is the $[n-1 / p]$ Padé approximant of $\widehat{\mathcal{E}}_{b}(z)$. This is indeed the case because we recognize $Q(z)$ in $(3.4)$ as being of the form given in [9] and unicity of Padé approximant enables us to conclude.

Proof of $(i i)$. This is simply a summary of what was proved in $(i)$. We have

$$
\widehat{\mathcal{E}}_{b}(z)=\sum_{k=0}^{n-1}(b)_{k} z^{k}+\left.(b)_{n} z^{n}\left([p / p]_{\Psi_{z}(t)}\right)\right|_{t=1 /(b-1+n)}+O\left(z^{n+p}\right)
$$

and the bounds for the degrees show that

$$
\sum_{k=0}^{n-1}(b)_{k} z^{k}+(b)_{n} z^{n}\left([p / p]_{\Psi_{z}(t)}\right)_{\mid t=1 /(b-1+n)}=[n-1 / p]_{\widehat{\mathcal{E}}_{b}(z)} .
$$


The above results are a priori proved under the assumption that $\Re(b)>0$. They hold under the weaker assumption that $b \in \mathbb{C} \backslash \mathbb{Z}_{\leq 0}$ because all these identities between rational fractions extend to this case.

\section{REFERENCES}

[1] Y. André, Séries Gevrey de type arithmétique, I. Théorèmes de pureté et de dualité, Annals of Math. 151 (2000), 705-740.

[2] R. ApÉRY, Irrationality of $\zeta(2)$ and $\zeta(3)$, Astérisque 61 (1979), 11-13.

[3] J. Arvesú, J. Coussement and W. Van Assche, Some discrete multiple orthogonal polynomials, J. Comput. Appl. Math. 153, 1-2 (2003), 19-45.

[4] P. D. Miller, Applied asymptotic analysis, Graduate Studies in Mathematics 75. American Mathematical Society, Providence, RI, 2006.

[5] M. PrÉvost, A new proof of the irrationality of $\zeta(2)$ and $\zeta(3)$ using Padé approximants, J. Comput. Appl. Math. 67.2 (1996), 219-235.

[6] M. Prévost and T. Rivoal, Remainder Padé approximants for the exponential function, Constr. Approx. 25.1 (2007), 109-123.

[7] T. Rivoal, Nombres d'Euler, approximants de Padé et constante de Catalan, Ramanujan J. 11.2 (2006), 199-214.

[8] T. Rivoal, On the arithmetic nature of the values of the Gamma function, Euler's constant and Gompertz's constant, Michigan Math. J. 61 (2012), 239-254.

[9] A. SIDI, A new method for deriving Padé approximants for some hypergeometric functions, J. Comput. Appl. Math. 7.1 (1981), 37-40.

[10] J. Touchard, Nombres exponentiels et nombres de Bernoulli, Canadian J. Math. 8 (1956), 305-320.

M. Prévost, lmpa Univ. Littoral Côte d’Opale, EA 2597- LMPA- Laboratoire de Mathématiques Pures et Apppliquées Joseph liouville, 62228 Calais, France, And CNRS, FR 2956, FRANCE

E-mail address: marc.prevost@univ-littoral.fr

T. Rivoal, Institut Fourier, CNRS et Université Grenoble Alpes, CS 40700, 38058 Grenoble cedex 9, France. 